\title{
氮素调控棉花纤维蔗糖代谢及纤维比强度的生理机制
}

\author{
马溶慧许乃银张传喜 李文峰冯营屈否王友华 周治国*
}

(南京农业大学 / 农业部作物生长调控重点开放实验室, 江苏南京 210095)

摘 要: 以棉纤维比强度高(科棉 1 号, 平均比强度为 $35 \mathrm{cN} \mathrm{tex}^{-1}$ )和中等(美棉 $33 \mathrm{~B}$, 平均比强度为 $32 \mathrm{cN} \mathrm{tex}^{-1}$ )的 2 个 基因型为材料, 于2005年在江苏省农业科学院(长江流域下游棉区)和江苏省䂙州县宿羊山镇(黄河流域黄淮棉区)设置 氮素水平(零氮为 $0 \mathrm{~kg} \mathrm{~h}^{-2}$, 适氮为 $240 \mathrm{~kg} \mathrm{hm}^{-2}$, 高氮为 $480 \mathrm{~kg} \mathrm{~h}^{-2}$ ) 试验, 研究氮素调控棉纤维蔗糖代谢及纤维比 强度的生理机制。结果表明, 棉铃对位叶氮浓度随铃龄变化的趋势符合幂函数曲线 $\left[Y_{\mathrm{N}}=a t^{-b}, Y_{\mathrm{N}}\right.$ 为棉铃对位叶氮浓 度 $(\%), t$ 为铃龄 $(\mathrm{d}), a 、 b$ 为参数]。高氮水平下的 $a$ 值显著增加, 导致铃龄 $24 \mathrm{~d}$ 前纤维中蔗糖代谢相关酶(蔗糖酶、蔗 糖合成酶和磷酸熫糖合成酶)活性和蔗糖转化量、纤维素最大累积速率以及铃龄 $24 \mathrm{~d}$ 纤维比强度降低; 零氮水平下的 $b$ 值显著增加, 与铃龄 $24 \mathrm{~d}$ 后纤维蔗糖代谢相关酶活性和蔗糖含量峰值降低、纤维素快速累积持续期缩短以及铃龄 $24 \mathrm{~d}$ 后纤维比强度增幅减小的关系密切。上述变化特征在品种间一致, 是棉纤维发育对棉铃对位叶氮浓度做出的重 要生理响应, 进而导致高氮、零氮水平下的成熟纤维比强度显著降低。铃龄 $24 \mathrm{~d}$ 是氮素调控棉纤维蔗糖代谢及纤维 比强度的转折期, 该时期的棉铃对位叶氮浓度分别为 3.15\% (南京)和 $2.75 \%$ (徐州)时有利于高强纤维的形成。

关键词：棉花 (Gossypium hirsutum L.)；棉铃对位叶；叶氮浓度；棉纤维；蔗糖代谢；纤维强比度

\section{Physiological Mechanism of Sucrose Metabolism in Cotton Fiber and Fiber Strength Regulated by Nitrogen}

MA Rong-Hui, XU Nai-Yin, ZHANG Chuan-Xi, LI Wen-Feng, FENG Ying, QU Lei, WANG You-Hua, and ZHOU Zhi-Guo*

(Key Laboratory of Crop Growth Regulation / Ministry of Agriculture, Nanjing Agricultural University, Nanjing 210095, Jiangsu, China)

Abstract: Fiber strength is one of the important criteria of cotton (Gossypium hirsutum L.) quality. Two cotton cultivars (KC-1,

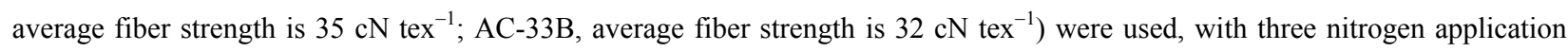
rates $\left(0,240,480 \mathrm{~kg} \mathrm{ha}^{-1}\right)$, standing for low, moderate and high nitrogen levels respectively in field experiments in Nanjing $\left(118^{\circ} 50^{\prime} \mathrm{E}, 32^{\circ} 02^{\prime} \mathrm{N}\right.$, middle lower reaches of Yangtze River Valley) and Xuzhou $\left(117^{\circ} 11^{\prime} \mathrm{E}, 34^{\circ} 15^{\prime} \mathrm{N}\right.$, Yellow River Valley), Jiangsu province, which stand for the different ecological conditions. The results showed that the changes of nitrogen concentration in the subtending leaf of cotton boll followed the equation: $Y_{\mathrm{N}}=a t^{-b} \quad\left[Y_{\mathrm{N}}\right.$ is nitrogen concentration in the subtending leaf of cotton boll (\%); $t$ is boll age (d); $a$ and $b$ are the parameters]. " $a$ " was significantly high under high-nitrogen level, which, to a great extent, led to the decreases of sucrose inversion amount and activity of the enzymes (invertase, sucrose synthetase, and phosphate sucrose synthetase) before the 24th day post anthesis (DPA), as well as the maximal speed of cellulose accumulation in cotton fiber and fiber strength at the 24th DPA. " $b$ " was significantly high under low-nitrogen level, which made negative effects on sucrose metabolism after the 24th DPA, shorten the duration for cellulose rapid accumulation in cotton fiber and reduced the increment of fiber strength from the 24th DPA to boll opening. The changes under high or low-nitrogen levels described above were important physiological responses of cotton fiber development to nitrogen concentration in the subtending leaf of cotton boll, and ultimately resulted in lower final fiber strength, and the changes showed similar trends in $\mathrm{KC}-1$ and AC-33B. The results in-

基金项目: 国家自然科学基金项目(30771277, 30771279); 公益性行业(农业)科研专项(nyhyzx07-005-18)

作者简介: 马溶慧(1979-), 女, 河南周口人, 博士研究生, 主要从事作物生理生态基础研究。

“通讯作者(Corresponding author): 周治国，教授，博士生导师。Tel: 025-84396813; E-mail: giscott@njau.edu.cn Received(收稿日期): 2008-04-24; Accepted(接受日期): 2008-07-15. 
dicated that, in the subtending leaf of cotton boll, the 24th DPA was a transition point of sucrose metabolism in cotton fiber and fiber strength regulated by nitrogen.

Keywords: Cotton; Subtending leaf of cotton boll; Leaf nitrogen concentration; Cotton fiber; Sucrose metabolism; Fiber strength

氮素是作物生产中最活跃、最难调控的因子之 一, 有关氮素对成熟棉纤维品质指标影响的研究已 有较多报道 ${ }^{[1-5]}$, 但因土壤质地的差异以及受化学氮 在土壤中流失、转化等因素的影响, 结论不尽一致, 有人认为适宜施氮可以改善棉纤维品质 ${ }^{[1-3]}$, 有人认 为氮素对棉纤维品质的影响较小 ${ }^{[4-5]}$ 。

叶片氮浓度是表征作物氮素营养水平的最直接 指标, 与表观的大田施氮量相比, 其高低与作物产 量和品质的关系更为密切, 是量化氮素影响的主要 指标 ${ }^{[6-9]}$ 。棉铃对位叶与棉铃在光合产物的合成、运 输和分配等方面表现密切的“源”与“库”关系 ${ }^{[10-11]}$, 棉铃对位叶氮浓度关系到叶片中光合产物的合成能 力及向棉铃运转的效率, 进而影响棉铃发育 ${ }^{[1]}$ 。对 高产棉花适宜叶片氮浓度的研究表明, 盛花期和吐 絮期的果枝叶氮浓度过高、过低均不利于产量形成 ${ }^{[12]}$, 而棉铃对位叶氮浓度如何调控棉纤维品质的形成, 是否存在适宜的叶片氮浓度尚未见报道。

纤维比强度是衡量棉纤维品质的重要指标之一, 主要取决于纤维加厚发育过程中纤维素的累积特 性 ${ }^{[13-14]}$ 。蔗糖是纤维素合成的初始底物, 决定纤维 素合成直接底物——尿苷二磷酸-D-葡萄糖(UDPG) 生成速率, 但该过程受多个酶调控。研究表明, 棉纤 维中的熫糖, 一部分被蔗糖合成酶分解成 UDPG 和 果糖, 为纤维素的合成提供直接底物, 另一部分则 被蔗糖酶水解为葡萄糖和果糖, 为纤维素的快速累 积提供碳源和能量 ${ }^{[15-16]}$ 。蔗糖合成酶是棉纤维发育 中的关键酶, 直接影响纤维素的合成速度和沉积质 量 ${ }^{[15-17]}$, 蔗糖酶则在纤维韧皮部的蔗糖卸载及液泡 中蔗糖浓度的调节过程中起重要作用 ${ }^{[18]}$ 。纤维中除 了蔗糖合成酶催化产生的 UDPG 外, 还存在大量不 能直接作为纤维素合成前体的游离态 UDPG, 被磷 酸庶糖合成酶催化继续为纤维素合成提供底物 ${ }^{[16,19]}$, 因此磷酸熫糖合成酶活性亦影响纤维素的沉积 ${ }^{[20]}$ 。 蔗糖代谢与作物产量、品质形成的关系密切, 在其他 作物上的研究表明, 该过程易受氮素水平的调控 ${ }^{[21-23]}$, 但有关氮素调控棉纤维中蔗糖代谢的研究尚未见报 道。

棉铃对位叶是棉铃发育的主要“源”, 其氮浓度
直接影响叶片中光合产物的合成与运输, 蔗糖是光 合产物运输的主要形式 ${ }^{[24}$, 转运到纤维中的蔗糖对 纤维品质形成起着关键作用 ${ }^{[15-16]}$, 但有关棉铃对位 叶氮浓度与纤维发育过程中的蔗糖代谢及纤维品质 形成之间的关系研究较少。因此, 本研究拟通过在 不同生态棉区设置氮肥试验, 研究棉铃对位叶氮浓 度、棉纤维加厚发育过程中蔗糖含量、蔗糖代谢相 关酶活性、纤维素累积和纤维比强度对氮素水平的 响应特征, 探索棉铃对位叶氮浓度与纤维中蔗糖代 谢及纤维比强度形成的关系, 以及氮素调控棉纤维 比强度形成的生理机制, 以期为量化研究棉纤维品 质的氮素营养监测提供理论依据。

\section{1 材料与方法}

\section{1 试验设计}

为兼顾试验的重复性和生态区域性, 试验于 2005 年在江苏省农业科学院(长江流域下游棉区)、 江苏省䂙州县宿羊山镇(黄河流域黄淮棉区)同时进 行, 供试土壤为壤土和黏土, $0 \sim 20 \mathrm{~cm}$ 土层分别含有 机质 $1.73 \% 、 1.47 \%$, 全氮 $0.11 \% 、 0.09 \%$, 碱解氮 $51.71 \mathrm{mg} \mathrm{kg}^{-1} 、 45.76 \mathrm{mg} \mathrm{kg}^{-1}$, 速效磷 $27.82 \mathrm{mg} \mathrm{kg}^{-1}$ 、 $21.34 \mathrm{mg} \mathrm{kg}^{-1}$, 速效钾 $103.27 \mathrm{mg} \mathrm{kg}^{-1} 、 89.26 \mathrm{mg}$ $\mathrm{kg}^{-1}$ 。棉纤维发育期内的总日照时数、日均温、总降 水量南京分别为 $234 \mathrm{~h} 、 26.6^{\circ} \mathrm{C} 、 312.8 \mathrm{~mm}$, 徐州分 别为 $207 \mathrm{~h} 、 25.2^{\circ} \mathrm{C} 、 421.3 \mathrm{~mm}$ 。

以科棉 1 号 (高强纤维品种, 平均比强度为 $35 \mathrm{cN}$ $\mathrm{tex}^{-1}$, 用 $\mathrm{KC}-1$ 表示)、美棉 $33 \mathrm{~B}$ (中强纤维品种, 平 均比强度为 $32 \mathrm{cN} \mathrm{tex}^{-1}$, 用 AC-33B 表示)为供试材 料, 根据薛晓萍等研究 ${ }^{[12]}, 240 \mathrm{~kg} \mathrm{hm}^{-2}$ 是棉花高产 适宜施纯氮量, 故氮素处理均设 $0 \mathrm{~kg} \mathrm{hm}^{-2}$ (N0)、240 $\mathrm{kg} \mathrm{hm}^{-2}$ (N240)和 $480 \mathrm{~kg} \mathrm{hm}^{-2}$ (N480) 3 个氮素水平, 分别代表零氮、适氮和高氮水平。所用氮肥为尿素, 基施 $50 \%$ 、花铃肥(始花期施用) $50 \%$ 。试验采取裂 区设计, 以品种为主区, 氮素为副区, 共 6 个处理, 小区面积均为 $9 \mathrm{~m} \times 6 \mathrm{~m}, 4$ 次重复。于 4 月 25 日营 养钵育苗, 5 月 18 日移栽, 行距 $0.75 \mathrm{~m}$, 株距 0.25 $\mathrm{m}$ 。为确保氮肥施用均匀, 基施氮肥后至移栽后第 2 天, 在棉行内相邻两棵棉苗之间打一个 $20 \mathrm{~cm}$ 深孔, 
每孔内定量施入设计数量的尿素。7月 8 日同样打孔定 量施入花铃肥。田间其他管理均按高产栽培要求进 行。

在棉株第 6 8 果枝第 $1 、 2$ 果节开花时，挂牌标 记当日内所开白花。对挂牌标记铃, 从铃龄 $10 \mathrm{~d}$ 开 始直至吐絮, 每隔 $7 \mathrm{~d}$ 在 9:00-10:00 取生长发育一 致的棉铃 8 10 个及其对位叶。将棉铃中的纤维与种 子分离后, 纤维样品用铌子混匀分成两份, 一份经 液氮速冻后于 $-30^{\circ} \mathrm{C}$ 保存供测定酶活用, 另一份经 风干(尽温 $>30^{\circ} \mathrm{C}$ )后用于测定蔗糖、纤维素含量和 3.2 $\mathrm{mm}$ 隔距纤维比强度。剪取棉铃对位叶中间部分, 洗 净擦干后 $105^{\circ} \mathrm{C}$ 杀青 $15 \mathrm{~min}$, 在 $70^{\circ} \mathrm{C}$ 下烘干至恒重, 供测试叶片氮浓度。待吐絮时，收取大小一致的挂 牌标记棉铃 20 个左右, 风干轧花后测定 $3.2 \mathrm{~mm}$ 隔 距纤维比强度。

1.2 测定项目与方法

\subsection{1 净光合速率 $\left(P_{\mathrm{n}}\right)$ 采用 LI-6400 (美国} LI-COR 公司)便携式光合仪, 对铃龄 $10 \mathrm{~d}$ 和 $40 \mathrm{~d}$ 挂 牌标记的棉铃对位叶在上午 9:00-11:00 进行测定, 使用开放式气路, 内置光源(光强为 $1500 \mu \mathrm{mol} \mathrm{s}{ }^{-1}$ $\mathrm{m}^{-2}$ ), 每个处理重复测定 5 次, 每张叶片测定 3 次取 其平均值。

\subsection{2 氮浓度粉碎已烘干的棉铃对位叶样品,} 过 40 目篮, 用凯氏定氮法测定叶片全氮含量并计算 其氮浓度 $(\%)^{[25]}$ 。
1.2.3蔗糖及蔗糖代谢相关酶活性用间苯二酚 显色法测定 ${ }^{[26]}$ 熫糖含量, DNS 显色法测定 ${ }^{[26]}$ 蔗糖酶 活性, 果糖和 UDPG 比色法测定 ${ }^{[26]}$ 磷酸庶糖合成酶 活性, 蔗糖和 UDP 比色法测定 ${ }^{[27]}$ 蔗糖合成酶活性。 1.2.4 纤维素含量 用瑞典产 1020 型半自动纤维 分析仪测定纤维素含量。

1.2.5 纤维比强度 将纤维样品混匀后用棉花纤 维引伸器制成棉条, 用国产 Y162 型束纤维强力机 测定 $3.2 \mathrm{~mm}$ 隔距比强度, 测 6 个重复, 取平均值作 为试样代表值, 并用中国纤维检验局的标准棉样修 正。

\section{3 统计分析}

采用 Excel 软件完成全部数据处理和作图, SPSS 11.5 统计软件进行分析。

\section{2 结果与分析}

\section{1 棉铃对位叶氮浓度变化特征}

两品种的棉铃对位叶氮浓度对氮素水平的响应 均呈下降趋势, 符合幂函数方程 $Y_{\mathrm{N}}=a t^{-b}(a 、 b$ 为参 数), 拟合度均达极显著水平, 试点间表现一致(表 1)。分析表 1 可知, 氮素水平显著影响方程的参数, $a$ 、 $b$ 值随氮素水平的变化趋势在品种和试点间趋于一 致, $a$ 值表现为 $\mathrm{N} 0$ 与 $\mathrm{N} 240$ 差异较小, 均显著低于 $\mathrm{N} 480 ; b$ 值均表现为随氮素水平提高而显著降低, 但 $\mathrm{N} 240$ 与 $\mathrm{N} 480$ 差异较小。

表 1 棉铃对位叶氮浓度变化特征对氮素水平的响应

Table 1 Response of changing characteristics of nitrogen concentration in the subtending leaf of cotton boll to nitrogen application rates

\begin{tabular}{|c|c|c|c|c|c|c|c|}
\hline \multirow{2}{*}{$\begin{array}{l}\text { 品种 } \\
\text { Cultivar }\end{array}$} & \multirow{2}{*}{$\begin{array}{c}\text { 氮素水平 } \\
\text { Nitrogen rate }\end{array}$} & \multicolumn{3}{|c|}{ 江苏南京 Nanjing, Jiangsu } & \multicolumn{3}{|c|}{ 江苏徐州 Xuzhou, Jiangsu } \\
\hline & & 方程 Equation & $R^{2}$ & $S_{e}$ & 方程 Equation & $R^{2}$ & $S_{e}$ \\
\hline \multirow{3}{*}{$\begin{array}{l}\text { 科棉 } 1 \text { 号 } \\
\mathrm{KC}-1\end{array}$} & $0 \mathrm{~kg} \mathrm{hm}^{-2}$ & $Y_{\mathrm{N}}=5.2321 t^{-0.2209}$ & $0.992^{* *}$ & 0.032 & $Y_{\mathrm{N}}=4.5001 t^{-0.2183}$ & $0.958^{* *}$ & 0.052 \\
\hline & $240 \mathrm{~kg} \mathrm{hm}^{-2}$ & $Y_{\mathrm{N}}=5.3507 t^{-0.1749}$ & $0.986^{* *}$ & 0.023 & $Y_{\mathrm{N}}=4.4838 t^{-0.1569}$ & $0.970^{* *}$ & 0.068 \\
\hline & $480 \mathrm{~kg} \mathrm{hm}^{-2}$ & $Y_{\mathrm{N}}=5.8346 t^{-0.1622}$ & $0.984^{* *}$ & 0.047 & $Y_{\mathrm{N}}=4.9821 t^{-0.1367}$ & $0.972^{* *}$ & 0.063 \\
\hline \multirow{3}{*}{$\begin{array}{l}\text { 美棉 33B } \\
\text { AC-33B }\end{array}$} & $0 \mathrm{~kg} \mathrm{hm}^{-2}$ & $Y_{\mathrm{N}}=5.8835 t^{-0.2469}$ & $0.979^{* *}$ & 0.045 & $Y_{\mathrm{N}}=4.9703 t^{-0.2425}$ & $0.993^{* *}$ & 0.052 \\
\hline & $240 \mathrm{~kg} \mathrm{hm}^{-2}$ & $Y_{\mathrm{N}}=6.0371 t^{-0.1961}$ & $0.976^{* *}$ & 0.094 & $Y_{\mathrm{N}}=5.0114 t^{-0.1810}$ & $0.983^{* *}$ & 0.049 \\
\hline & $480 \mathrm{~kg} \mathrm{hm}^{-2}$ & $Y_{\mathrm{N}}=6.5146 t^{-0.1761}$ & $0.993^{* *}$ & 0.050 & $Y_{\mathrm{N}}=5.5687 t^{-0.1655}$ & $0.972^{* *}$ & 0.084 \\
\hline
\end{tabular}

$Y_{\mathrm{N}}$ : 棉铃对位叶氮浓度 $(\%) ; t$ : 铃龄 $(\mathrm{d}){ }^{*}{ }^{* *}$ : 方程决定系数在 0.01 水平上显著 $\left(n=6, P_{0.01}=0.8413\right)$ 。

$Y_{\mathrm{N}}$ : Nitrogen concentration in the subtending leaf of cotton boll (\%); $t$ : boll age (d); ${ }^{* *}$ : significance of determination coefficient at the 0.01 probability level $\left(n=6, P_{0.01}=0.8413\right)$.

\section{2 棉铃对位叶净光合速率 $\left(P_{n}\right)$}

如图 1 所示, 2 个品种在两试点的棉铃对位叶 $P_{\mathrm{n}}$ 对氮素水平的响应趋于一致, N0、N480 在铃龄 $10 \mathrm{~d}$ 的 $P_{\mathrm{n}}$ 较 N240 分别降低 $13.2 \% 、 6.2 \%$; 铃龄 $40 \mathrm{~d}$ 时
N0 较 N240 降低 $40.1 \%, \mathrm{~N} 480$ 则增加 $9.7 \%$ 。说明零 氮显著降低了棉铃对位叶的光合能力, 且随着棉铃 发育进程的推移其降幅逐渐加大; 而高氮虽降低了 棉铃发育前期的 $P_{\mathrm{n}}$ ，但有利于提高后期的 $P_{\mathrm{n}}$ 。 

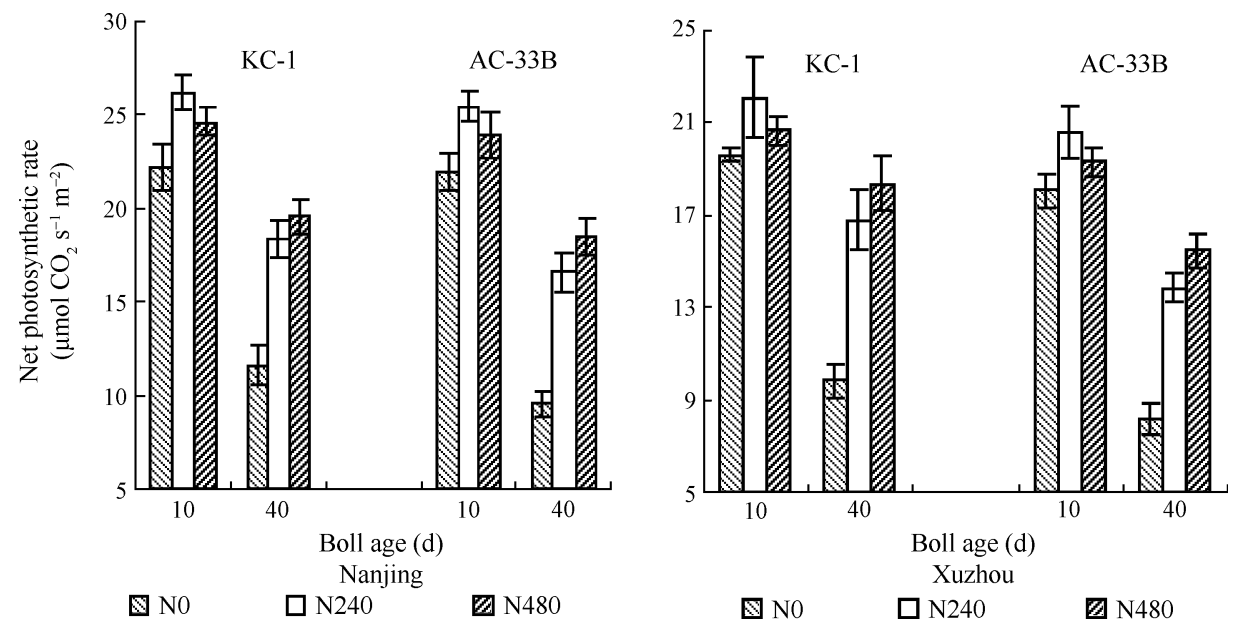

图 1 棉铃对位叶净光合速率对氮素水平的响应

Fig. 1 Response of net photosynthetic rate in the subtending leaf of cotton boll to nitrogen application rates

2.3 棉纤维中蔗糖代谢相关酶活性变化

2.3.1 蔗糖酶(invertase, Inv) 由图 2 可见, 2 个品
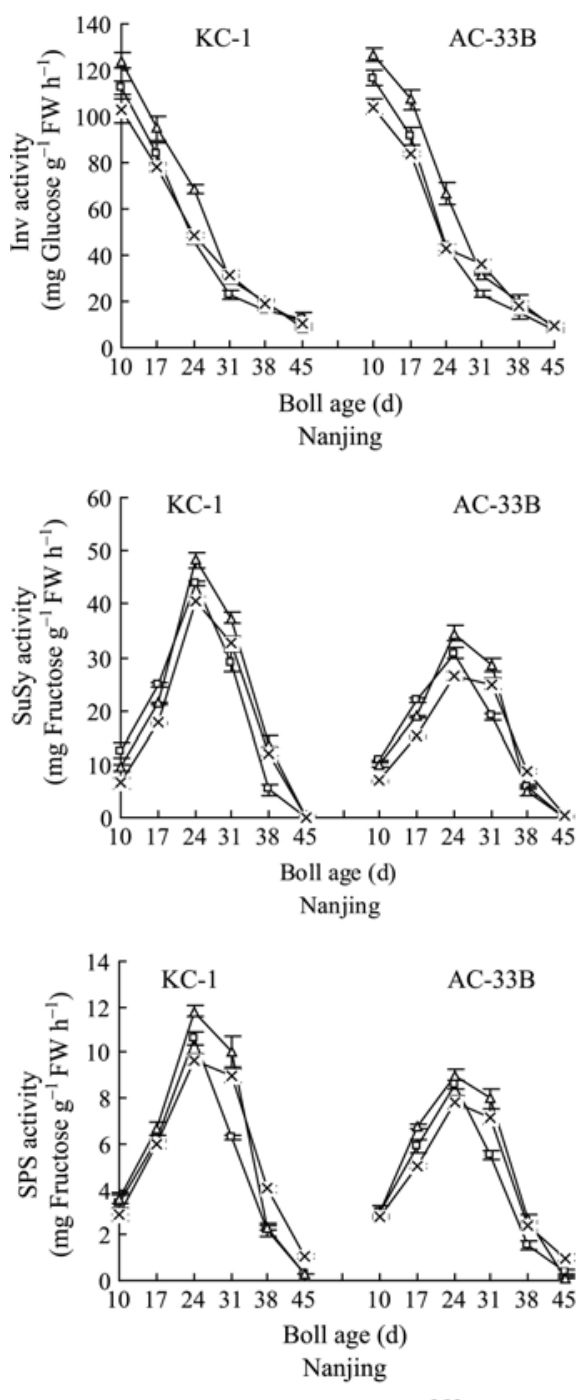

$$
\rightarrow \text { N0 } \rightarrow \text { N240 }
$$

种的棉纤维 Inv 活性随铃龄均呈下降趋势, 2 个试点 的试验结果一致。处理间差异主要表现在酶活性的
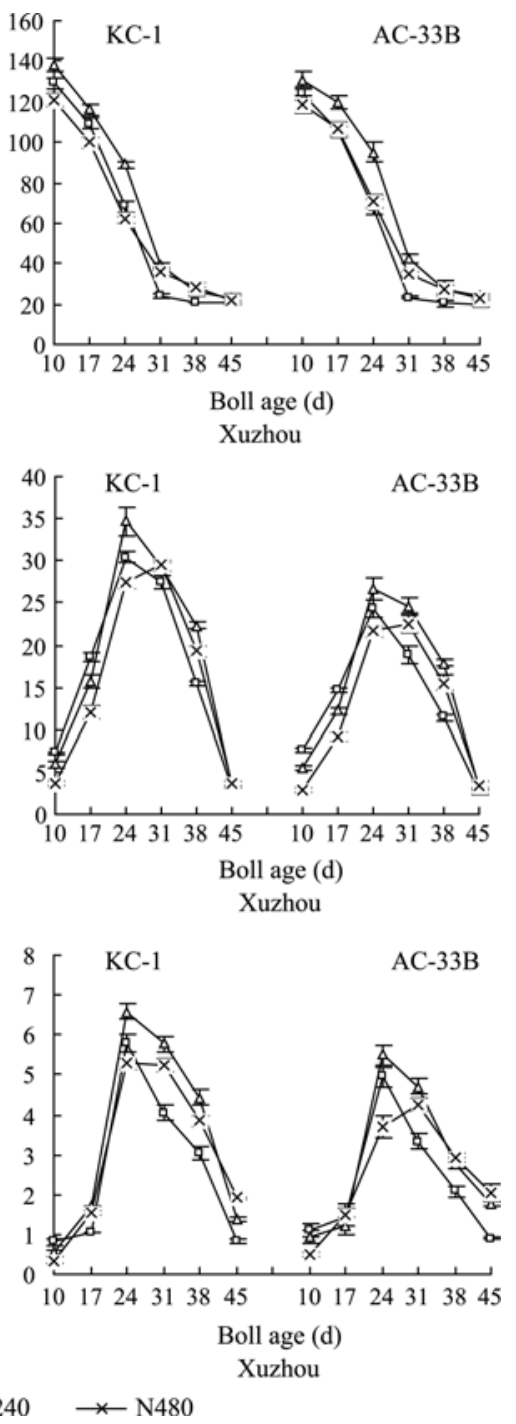

图 2 棉纤维中蔗糖代谢相关酶活性变化对氮素水平的响应

Fig. 2 Response of dynamic changes for enzymes activities associated with sucrose metabolism in cotton fiber to nitrogen application rates 
高低上, 铃龄 $31 d$ 前 N240 的 Inv 活性显著高于 N0 和 N480, 之后处理间差异缩小且均维持较低水平。 说明适氮水平下棉纤维中 Inv 可维持较长时间的高 活性, 有利于熫糖的水解, 为纤维素快速累积提供 较多的碳源和能量。

\subsection{2 蔗糖合成酶(sucrose synthetase, SuSy) 棉纤} 维 SuSy 活性随铃龄的变化呈单峰曲线(图 2), 峰值出 现在铃龄 $24 \mathrm{~d}$ 左右。2 个品种间差异表现为科棉 1 号 高于美棉 33B, 但对氮素水平的响应趋势在试点间一 致。以 N240 的 SuSy 活性峰值最高, 峰值前随氮素水 平提高而降低, 峰值后 $\mathrm{N} 240$ 与 $\mathrm{N} 480$ 间差异较小, 均 高于 $\mathrm{N} 0$ 。说明适氮水平下 SuSy 活性较高, 有利于分 解较多的蔗糖, 为纤维素合成提供较多的底物 UDPG。

2.3.3 磷酸庶糖合成酶(sucrose phosphate synthetase, SPS) 如图 2 所示, 棉纤维 SPS 活性的变化与 SuSy

相似, 处理间差异主要表现在 SPS 活性高低上, 铃 龄 $24 \mathrm{~d}$ 时以 $\mathrm{N} 240$ 最高, 之后 $\mathrm{N} 240$ 与 $\mathrm{N} 480$ 间差异 较小, 均高于 $\mathrm{N} 0$ 。说明适氮水平可提高纤维中 SPS 活性, 相应增加了为纤维素合成提供的物质基础和 能量。

\section{4 棉纤维中蔗糖和纤维素含量的变化}

2.4.1 蔗糖含量 如图 3 所示, 2 个品种的棉纤维 中熫糖含量随铃龄的变化呈下降趋势, 在铃龄 24 31 d 期间下降较慢或稍微上升，该趋势在试点间 一致。铃龄 $10 \mathrm{~d}$ 时熫糖含量、铃龄 10 24d 期间熫 糖含量减少量均表现为 $\mathrm{N} 0$ 与 $\mathrm{N} 240$ 差异较小, 显著 高于 $\mathrm{N} 480 ; \mathrm{N} 240$ 在铃龄 $31 \mathrm{~d}$ 时蔗糖含量与 $\mathrm{N} 480$ 差 异较小, 均显著高于 $\mathrm{N} 0$ 。说明适氮水平有利于纤维 中蔗糖的转化和积累, 这与蔗糖代谢相关酶活性对 氮素水平响应的结果一致。
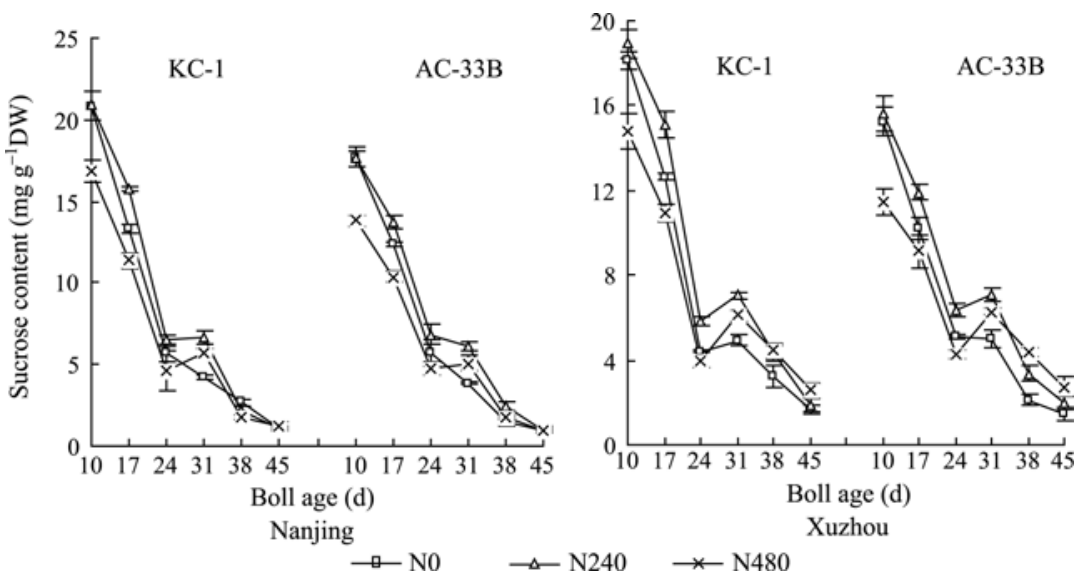

图 3 棉纤维中蔗糖含量动态变化对氮素水平的响应

Fig. 3 Response of dynamic changes of sucrose content in cotton fiber to nitrogen application rates

\section{4 .2 纤维素含量 棉纤维素含量的变化呈“S”} 型曲线，可用 Logistic 模型对其拟合，拟合方程均达 极显著水平(表 2)。当纤维素含量达到 $80 \%$ 以上时， 纤维比强度的差异主要由纤维素最大累积速率 $\left(V_{\max }\right)$ 和快速累积持续期 $(T)$ 决定 ${ }^{[14]}$ 。如表 2 所示, 2 个品种 的 $V_{\max }$ 值、 $T$ 值对氮素水平的响应趋势在试点间一 致。 $V_{\max }$ 值以 $\mathrm{N} 480$ 最小, 较 $\mathrm{N} 0 、 \mathrm{~N} 240$ 分别降低了 $20.8 \% 、 16.6 \%, T$ 值则以 N0 最小, 较 N240、N480 分别缩短了 $2 \mathrm{~d}$ 和 $3 \mathrm{~d}$ 。说明适氮水平下的纤维素含 量具有较大的累积速率和较长的快速累积期, 而高 氮、零氮水平分别降低了纤维素累积速率、缩短了 纤维素快速累积期。综合分析图 3 和表 2 发现, 铃 龄 $24 \mathrm{~d}$ 之前的蔗糖转化量越多, $V_{\max }$ 值越大; 铃龄 $24 \mathrm{~d}$ 之后蔗糖含量峰值越高, $T$ 值越大。
2.5 棉纤比强度的变化

如图 4 所示, 铃龄 $24 \mathrm{~d}$ 后的纤维比强度随铃龄 呈持续上升趋势。2 2 个品种间差异表现为科棉 1 号 高于美棉 33B，但对氮素水平的响应趋势在试点间 一致。铃龄 $24 \mathrm{~d}$ 的纤维比强度 $\mathrm{N} 0$ 与 $\mathrm{N} 240$ 差异较小, 均显著高于 $\mathrm{N} 480$; 铃龄 $24 \mathrm{~d}$ 至吐絮期间纤维比强度 增加量 $\mathrm{N} 240$ 与 $\mathrm{N} 480$ 差异较小, 均显著高于 $\mathrm{N} 0$; 成 熟纤维比强度 N240 显著高于 N480、N0。说明适氮 水平下铃龄 $24 \mathrm{~d}$ 的纤维比强度较高, 且之后的增幅 较大, 有利于形成较高的成熟纤维比强度。

2.6 棉铃对位叶氮浓度与纤维中蔗糖、纤维素含 量及纤维比强度之间的关系

综合上述分析结果可知，高氮、零氮均显著影 响了棉铃对位叶氮浓度随铃龄变化回归方程的系数, 
表 2 棉纤维中纤维素含量累积特征对氮素水平的响应

Table 2 Response of characteristics of cellulose accumulation in cotton fiber to nitrogen application rates

\begin{tabular}{|c|c|c|c|c|c|c|}
\hline $\begin{array}{l}\text { 地点 } \\
\text { Site }\end{array}$ & $\begin{array}{c}\text { 品种 } \\
\text { Cultivar }\end{array}$ & $\begin{array}{c}\text { 氮素水平 } \\
\text { Nitrogen rate }\end{array}$ & $\begin{array}{l}\text { 模型 } \\
\text { Model }\end{array}$ & $R^{2}$ & $\begin{array}{c}V_{\max } \\
\left(\% \mathrm{~d}^{-1}\right)\end{array}$ & $\begin{array}{c}T \\
\text { (d) }\end{array}$ \\
\hline 江苏南京 & 科棉 1 号 & $0 \mathrm{~kg} \mathrm{hm}^{-2}$ & $Y_{\mathrm{c}}=83.1353 /\left(1+33.7356 \mathrm{e}^{-0.2125 t}\right)$ & $0.9852^{* *}$ & 4.4166 & 12 \\
\hline \multirow{5}{*}{$\begin{array}{l}\text { Nanjing, } \\
\text { Jiangsu }\end{array}$} & \multirow[t]{2}{*}{ KC-1 } & $240 \mathrm{~kg} \mathrm{hm}^{-2}$ & $Y_{\mathrm{c}}=93.0377 /\left(1+36.6883 \mathrm{e}^{-0.1897 t}\right)$ & $0.9611^{* *}$ & 4.4123 & 14 \\
\hline & & $480 \mathrm{~kg} \mathrm{hm}^{-2}$ & $Y_{c}=86.4844 /\left(1+25.5517 \mathrm{e}^{-0.1705 t}\right)$ & $0.9866^{* *}$ & 3.6864 & 15 \\
\hline & 美棉 33B & $0 \mathrm{~kg} \mathrm{hm}^{-2}$ & $Y_{\mathrm{c}}=80.5518 /\left(1+35.2119 \mathrm{e}^{-0.2096 t}\right)$ & $0.9592^{* *}$ & 4.2209 & 13 \\
\hline & \multirow[t]{2}{*}{$A C-33 B$} & $240 \mathrm{~kg} \mathrm{hm}^{-2}$ & $Y_{\mathrm{c}}=92.0010 /\left(1+33.2024 \mathrm{e}^{-0.1764 t}\right)$ & $0.9911^{* *}$ & 4.0572 & 15 \\
\hline & & $480 \mathrm{~kg} \mathrm{hm}^{-2}$ & $Y_{\mathrm{c}}=82.7851 /\left(1+24.9192 \mathrm{e}^{-0.1609 t}\right)$ & $0.9843^{* *}$ & 3.3300 & 16 \\
\hline \multirow{6}{*}{$\begin{array}{l}\text { 江苏徐州 } \\
\text { Xuzhou, } \\
\text { Jiangsu }\end{array}$} & \multirow{3}{*}{$\begin{array}{l}\text { 科棉 } 1 \text { 号 } \\
\mathrm{KC}-1\end{array}$} & $0 \mathrm{~kg} \mathrm{hm}^{-2}$ & $Y_{\mathrm{c}}=83.1588 /\left(1+33.9666 \mathrm{e}^{-0.2012 t}\right)$ & $0.9713^{* *}$ & 4.1829 & 13 \\
\hline & & $240 \mathrm{~kg} \mathrm{hm}^{-2}$ & $Y_{\mathrm{c}}=92.7429 /\left(1+34.4692 \mathrm{e}^{-0.1662 t}\right)$ & $0.9679^{* *}$ & 3.8535 & 16 \\
\hline & & $480 \mathrm{~kg} \mathrm{hm}^{-2}$ & $Y_{\mathrm{c}}=82.6566 /\left(1+36.8581 \mathrm{e}^{-0.1552 t}\right)$ & $0.9783^{* *}$ & 3.2071 & 17 \\
\hline & \multirow{3}{*}{$\begin{array}{l}\text { 美棉 33B } \\
\text { AC-33B }\end{array}$} & $0 \mathrm{~kg} \mathrm{hm}^{-2}$ & $Y_{\mathrm{c}}=80.8086 /\left(1+31.4154 \mathrm{e}^{-0.2036 t}\right)$ & $0.9851^{* *}$ & 4.1132 & 13 \\
\hline & & $240 \mathrm{~kg} \mathrm{hm}^{-2}$ & $Y_{\mathrm{c}}=90.4651 /\left(1+27.9684 \mathrm{e}^{-0.1658 t}\right)$ & $0.9920^{* *}$ & 3.7498 & 16 \\
\hline & & $480 \mathrm{~kg} \mathrm{hm}^{-2}$ & $Y_{\mathrm{c}}=81.5503 /\left(1+26.5541 \mathrm{e}^{-0.1561 t}\right)$ & $0.9812^{* *}$ & 3.1825 & 17 \\
\hline
\end{tabular}
续期。

$Y_{\mathrm{c}}$ : 纤维素含量 $(\%) ; t$ : 铃龄 $(\mathrm{d}) ; n=6, P_{0.01}=0.8413 ;{ }^{* *}$ : 在 0.01 水平上显著; $V_{\max }$ : 纤维素累积的最大速率; $T$ : 纤维素快速累积持

$Y_{\mathrm{c}}$ : cellulose content in cotton fiber (\%); $t$ : boll age (d); $n=6, P_{0.01}=0.8413 ;{ }^{* *}$ : significantly different at the 0.01 probability level; $V_{\max }$ : the maximal speed of cellulose accumulation in cotton fiber; $T$ : period for cellulose speedily accumulating in cotton fiber.
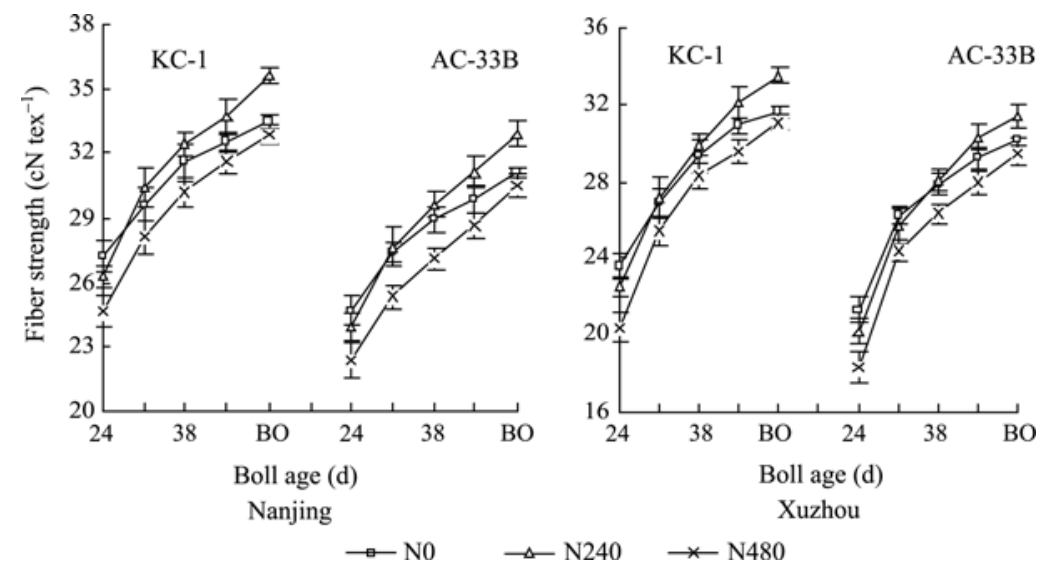

图 4 棉纤维比强度动态变化对氮素水平的响应

Fig. 4 Response of dynamic changes of cotton fiber strength to nitrogen rates BO: boll opening.

阻滞了纤维中蔗糖转化与积累的进程, 相应降低了 纤维素累积速率、缩短了纤维素快速累积期, 导致 成熟纤维比强度显著降低。

为了深入分析氮素水平对棉铃对位叶氮浓度、 蔗糖含量、纤维素累积及纤维比强度的影响, 以 N240 的上述各相应指标为对照, 计算 N0、N480 的 效应因子 $E I=(\mathrm{N} 240-\mathrm{N} 0$ 或 N480) $\times 100 / \mathrm{N} 240$ (表 3), 当 $E I>0$ 时为负效应, 当 $E I<0$ 时为正效应, 且 $E I$ 绝 对值越大, 其影响程度越大。

分析表 3 中效应因子的绝对值可知, N0 对 $b$ 、 $S_{31} 、 T$ 和 $S t r_{\text {in }}$ 的影响程度较大, N480 则对 $a 、 S_{\mathrm{de}}$ 、
$V_{\text {max }}$ 和 $S t r_{24}$ 的影响程度较大, 但 N0 与 N480 影响 $S t r_{\mathrm{BO}}$ 的差异较小。说明零氮与高氮水平对棉铃对位 叶氮浓度、纤维中熫糖、纤维素含量及纤维比强度 的影响程度存在较大差异。

进一步分析棉铃对位叶氮浓度效应因子绝对值与 棉纤维中蔗糖、纤维素含量及纤维比强度效应因子绝对 值之间相关性的结果表明(表 4), $a$ 值的效应因子绝对值 与 $S_{\mathrm{de}} 、 V_{\max }$ 和 $S t r_{24}$ 的效应因子绝对值之间呈显著或极 显著正相关，与 $S_{31} 、 T$ 和 $S t r$ in 的效应因子绝对值之间呈 极显著负相关; $b$ 值的效应因子绝对值与上述特征值效 应因子绝对值之间所呈现的相关性与 $a$ 值相反。 
表 3 氮素水平对棉铃对位叶氮浓度、纤维中蔗糖、纤维素含量及纤维比强度特征值影响的效应值

Table 3 The effect indices (EI) of nitrogen on eigen values of nitrogen concentration in the subtending leaf of cotton boll, sucrose, and cellulose content in cotton fiber and fiber strength

\begin{tabular}{|c|c|c|c|c|c|c|c|c|c|c|c|}
\hline $\begin{array}{l}\text { 地点 } \\
\text { Site }\end{array}$ & $\begin{array}{c}\text { 品种 } \\
\text { Cultivar }\end{array}$ & $\begin{array}{c}\text { 施氮量 } \\
\text { Nitrogen rate }\end{array}$ & $a$ & $b$ & $S_{\mathrm{de}}$ & $S_{31}$ & $V_{\max }$ & $T$ & $\mathrm{Str}_{24}$ & $S t r_{\text {in }}$ & $S t r_{\mathrm{BO}}$ \\
\hline \multirow{4}{*}{$\begin{array}{l}\text { 江苏南京 } \\
\text { Nanjing, } \\
\text { Jiangsu }\end{array}$} & \multirow{2}{*}{$\begin{array}{l}\text { 科棉 } 1 \text { 号 } \\
\mathrm{KC}-1\end{array}$} & $0 \mathrm{~kg} \mathrm{hm}^{-2}$ & -2.2 & 26.3 & 4.6 & -36.8 & 0.1 & -14.3 & 3.4 & -32.3 & -5.9 \\
\hline & & $480 \mathrm{~kg} \mathrm{hm}^{-2}$ & 9.0 & -7.3 & -15.4 & -14.9 & -16.5 & 7.1 & -6.5 & -10.8 & -7.6 \\
\hline & \multirow{2}{*}{$\begin{array}{l}\text { 美棉 33B } \\
\text { AC-33B }\end{array}$} & $0 \mathrm{~kg} \mathrm{hm}^{-2}$ & -2.5 & 25.9 & 9.2 & -36.8 & 4.0 & -13.3 & 3.2 & -28.5 & -5.5 \\
\hline & & $480 \mathrm{~kg} \mathrm{hm}^{-2}$ & 7.9 & -10.2 & -16.8 & -17.4 & -17.9 & 6.7 & -6.4 & -9.7 & -7.3 \\
\hline \multirow{4}{*}{$\begin{array}{l}\text { 江苏徐州 } \\
\text { Xuzhou, } \\
\text { Jiangsu }\end{array}$} & \multirow{2}{*}{$\begin{array}{l}\text { 科棉 } 1 \text { 号 } \\
\mathrm{KC}-1\end{array}$} & $0 \mathrm{~kg} \mathrm{hm}^{-2}$ & 0.4 & 39.1 & 4.4 & -29.9 & 8.6 & -18.8 & 4.9 & -27.3 & -5.7 \\
\hline & & $480 \mathrm{~kg} \mathrm{hm}^{-2}$ & 11.1 & -12.9 & -17.4 & -13.2 & -16.8 & 6.3 & -9.3 & -2.7 & -6.9 \\
\hline & \multirow{2}{*}{$\begin{array}{l}\text { 美棉 33B } \\
\text { AC-33B }\end{array}$} & $0 \mathrm{~kg} \mathrm{hm}^{-2}$ & -0.8 & 34.0 & 8.6 & -29.3 & 9.7 & -18.8 & 5.8 & -22.0 & -4.1 \\
\hline & & $480 \mathrm{~kg} \mathrm{hm}^{-2}$ & 11.1 & -8.6 & -22.8 & -11.3 & -15.1 & 6.3 & -9.2 & -1.2 & -5.4 \\
\hline
\end{tabular}

$a, b$ : 棉铃对位叶氮浓度随铃龄变化回归方程的参数; $S_{\mathrm{de}}$ ：铃龄 $10 \sim 24 \mathrm{~d}$ 期间蔗糖含量减少量; $S_{31}$ ：铃龄 $31 \mathrm{~d}$ 的蔗糖含量; $V_{\text {max }}$ ：纤 维素累积的最大速率; $T$ : 纤维素快速累积持续期; $\operatorname{Str}_{24}$ ：铃龄 $24 \mathrm{~d}$ 的纤维比强度; $\operatorname{Str}_{\mathrm{in}}$ ：铃龄 $24 \mathrm{~d}$ 至吐絮期间纤维比强度增加量; St $_{\mathrm{BO}}$ : 成熟纤维比强度。

$a, b$ : parameters in equation of nitrogen concentration dynamic changes along with boll age in the subtending leaf of cotton boll; $S_{\mathrm{de}}$ : the decrease of sucrose content in cotton fibers from the 10th to 24th DPA; $S_{31}$ : sucrose content in cotton fibers at the 31 st DPA; $V_{\max }$ : the maximal speed of cellulose accumulation in cotton fiber; $T$ : duration for cellulose speedily accumulating in cotton fiber; Str $r_{24}$ : fiber strength at the 24th DPA; Str in $_{\text {in }}$ the increment of fiber strength from the 24th DPA to boll opening; $S t r_{\mathrm{BO}}$ : final fiber strength.

表 4 效应因子绝对值之间的相关系数

Table 4 Correlation coefficients between the absolute value of effect indices

\begin{tabular}{cccccccc}
\hline & $S_{\mathrm{de}}$ & $S_{31}$ & $V_{\max }$ & $T$ & Str $_{24}$ & $\operatorname{Str}_{\text {in }}$ & Str $_{\mathrm{BO}}$ \\
\hline$a$ & $0.914^{* *}$ & $-0.920^{* *}$ & $0.793^{*}$ & $-0.942^{* *}$ & $0.860^{* *}$ & $-0.938^{* *}$ & 0.619 \\
$b$ & $-0.882^{* *}$ & $0.808^{*}$ & -0.703 & $0.979^{* *}$ & -0.634 & $0.818^{*}$ & -0.692 \\
\hline
\end{tabular}

${ }^{*}$ 和 ${ }^{* *}$ 分别表示在 0.05 和 0.01 水平上显著相关 $\left(n=8, P_{0.05}=0.707, P_{0.01}=0.834\right)$ 。缩写同表 3 。 Table 3.

" and ${ }^{* *}$ indicate significance at the 0.05 and 0.01 probability levels, respectively $\left(n=8, P_{0.05}=0.707, P_{0.01}=0.834\right)$. Abbreviations as in

综上所述, $a$ 值过大是导致铃龄 $24 \mathrm{~d}$ 前蔗糖转化 量、纤维素最大累积速率及铃龄 $24 \mathrm{~d}$ 纤维比强度降 低的重要原因, 而铃龄 $24 \mathrm{~d}$ 后蔗糖含量峰值的降 低、纤维素快速累积期的缩短及铃龄 $24 \mathrm{~d}$ 后纤维比 强度增幅的减小与 $b$ 值过大有密切关系。

\section{3 讨论}

叶片中的氮多数用于构建叶绿体, 参与光合作

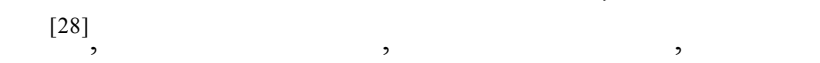
含量相应减少, 光合能力较低; 过量施氮虽能增加 叶绿素含量, 但叶片氮浓度过高, 造成叶片中氮代 谢过于旺盛, 增加了碳投入, 亦不利于发挥叶片的 光合能力 ${ }^{[1]}$ 。本研究结果表明, 氮素水平显著影响 棉铃对位叶氮浓度随铃变化回归方程的参数, 480 $\mathrm{kg} \mathrm{hm}^{-2}$ 处理的 $a$ 值最大, 其铃龄 $10 \mathrm{~d}$ 棉铃对位叶净 光合速率反而较 $240 \mathrm{~kg} \mathrm{hm}^{-2}$ 处理降低, 说明高氮水 平下棉铃对位叶氮浓度在棉铃发育初期过高; $0 \mathrm{~kg}$ $\mathrm{hm}^{-2}$ 处理的 $b$ 值最大, 其棉铃对位叶净光合速率较
$240 \mathrm{~kg} \mathrm{hm}^{-2}$ 处理降低的幅度随铃龄增长而增加, 由 此推断零氮水平下棉铃对位叶氮浓度在棉铃发育期 内的降幅过大。

近年来的研究结果已证明纤维素合成的直接底 物—UDPG 是由熫糖合成酶催化蔗糖降解提供 的 $^{[16-17]}$, 因此棉纤维中熫糖含量及其变化(蔗糖代谢 相关酶的调控)对纤维素累积具有重要影响 ${ }^{[24]}$ 。本研 究表明, 纤维中蔗糖含量和转化量对氮素水平的响 应趋势与蔗糖代谢相关酶活性保持高度一致, 铃龄 $24 \mathrm{~d}$ 前庶糖转化量越多, 纤维素最大累积速率则越 大; 铃龄 $24 \mathrm{~d}$ 后蔗糖含量峰值越高, 纤维素快速累 积期则越长。因此, 可以认为 $240 \mathrm{~kg} \mathrm{~h}^{-2}$ 处理的蔗 糖代谢相关酶维持长时间高活性, 是促进蔗糖向 UDPG 转化、合成更多纤维素的重要原因。当棉纤 维素含量达到 $80 \%$ 以上时, 最终纤维素含量对纤维 比强度的影响较小 ${ }^{[29]}$, 而纤维素最大累积速率和快 速累积持续期与纤维比强度的关系密切 ${ }^{[14,30]}$, 本研 究发现, $480 \mathrm{~kg} \mathrm{hm}^{-2}$ 处理的纤维素最大累积速率最 
低, $0 \mathrm{~kg} \mathrm{hm}^{-2}$ 处理的纤维素快速累积持续期最短, 相应地最终纤维比强度均较 $240 \mathrm{~kg} \mathrm{hm}^{-2}$ 处理显著降 低。因此, 可以推断在纤维素累积速率较高的基础 上有效延长纤维素的快速累积持续期, 有利于高强 纤维的形成。

棉铃发育过程中所需的营养物质大部分由棉铃 对位叶光合作用产生的碳水化合物提供 ${ }^{[12]}$, 主要以 庶糖形式输出到质外体并被装载到韧皮部, 再经韧 皮部运输到棉铃当中 ${ }^{[24]}$ 。适宜施氮可以改善棉花的 源库关系, 提高光合产物向生殖器官运转的效率; 高氮或缺氮均导致棉铃对位叶可溶性糖含量降低, 碳代谢迟缓, 不利于光合产物向棉铃运输 ${ }^{[11]}$ 。本研 究表明, $480 \mathrm{~kg} \mathrm{hm}^{-2}$ 处理的棉铃对位叶氮浓度在棉 铃发育初期过高, 降低了这时棉铃对位叶净光合速 率, 导致铃龄 $24 \mathrm{~d}$ 前庶糖代谢相关酶活性和蔗糖转 化量、纤维素最大累积速率以及铃龄 $24 \mathrm{~d}$ 的纤维比 强度显著降低。其原因可能在于氮浓度过高一方面 造成叶片中氮代谢过于旺盛 ${ }^{[28]}$, 另一方面也会加剧 棉田的郁蔽程度 ${ }^{[11]}$, 两者均不利于棉铃对位叶中碳 水化合物的积累和转运; $0 \mathrm{~kg} \mathrm{hm}^{-2}$ 处理的棉铃对位 叶氮浓度降幅过大, 降低了铃龄 $24 \mathrm{~d}$ 后蔗糖代谢的 强度, 缩短了纤维素的快速累积持续期, 不利于铃 龄 $24 \mathrm{~d}$ 后纤维比强度的增加。这主要由于氮浓度降 幅过大, 导致棉铃对位叶过早衰老 ${ }^{[28]}$, 进而降低了 铃龄 $24 \mathrm{~d}$ 后光合产物的合成与供应能力。

综上所述, $480 \mathrm{~kg} \mathrm{hm}^{-2}$ 处理的棉铃对位叶氮浓 度在棉铃发育初期过高, $0 \mathrm{~kg} \mathrm{hm}^{-2}$ 处理的棉铃对位 叶氮浓度在棉铃发育后期过低, 棉铃对位叶氮浓度 在铃龄 $24 \mathrm{~d}$ 前过高、铃龄 $24 \mathrm{~d}$ 后过低均不利于棉纤 维的庶糖代谢, 进而影响纤维素的累积方式和纤维 比强度。因此, 可以认为铃龄 $24 \mathrm{~d}$ 左右是棉铃对位 叶氮浓度调控纤维比强度的转折期。在本研究中, $240 \mathrm{~kg} \mathrm{hm}^{-2}$ 处理的纤维比强度最高, 其相应铃龄 24 $\mathrm{d}$ 的棉铃对位叶氮浓度在品种间差异较小, 而试点 间差异较大, 南京为 $3.15 \%$, 高于徐州 $2.75 \%$, 其原 因可能在于徐州试点的土壤质地为壤土, 较南京试 点的黏土保肥能力差, 加上徐州试点棉铃发育期内 的总降水量 $(421.3 \mathrm{~mm})$, 高于南京 $(312.8 \mathrm{~mm})$, 导致 土壤中的氮素流失较多, 棉株吸氮量下降, 棉铃对 位叶氮浓度随之降低。

\section{4 结论}

棉铃对位叶氮浓度随铃龄变化的趋势符合幂函
数方程 $Y_{\mathrm{N}}=a t^{-b}\left(Y_{\mathrm{N}}\right.$ : 棉铃对位叶氮浓度, $t$ : 铃龄, $a$, $b$ 为参数)。 $a$ 值过大与铃龄 $24 \mathrm{~d}$ 前纤维中蔗糖代谢 相关酶(庶糖酶、蔗糖合成酶和磷酸熫糖合成酶)活 性、蔗糖转化量、纤维素的最大累积速率和铃龄 24 $\mathrm{d}$ 纤维比强度的降低关系密切, $b$ 值过大则导致铃龄 $24 \mathrm{~d}$ 后熫糖代谢受阻, 纤维素快速累积持续期缩短, 纤维比强度下降, 其关系不受具体品种的影响。铃 龄 $24 \mathrm{~d}$ 是棉铃对位叶氮浓度调控纤维比强度的转折 期, 该时期适宜的棉铃对位叶氮浓度值分别为 $3.15 \%$ (南京)和 $2.75 \%$ (徐州)。

\section{References}

[1] Singh V, Nagwekar S N. Effect of weed control and nitrogen levels on quality characters in cotton. Indian Soc Cotton Improv, 1989, 14: 60-64

[2] Bauer P J, Roof M E. Nitrogen, aldicarb, and cover crop effects on cotton yield and fiber properties. Agron J, 2004, 96: 369-376

[3] Read J J, Reddy K R, Jenkins J N. Yield and fiber quality of upland cotton as influenced by nitrogen and potassium nutrition. Eur J Agron, 2006, 24: 282-290

[4] Bauer B J, Camberato J J, Roach S H. Cotton yield and fiber quality response to green manures and nitrogen. Agron $J$, 1993, 85: 1019-1023

[5] Sawan Z M, Mahmoud M H, Momtaz O A. Influence of nitrogen fertilization and foliar application of plant growth retardants and zinc on quantitative and qualitative properties of Egyptian cotton(Gossypium barbadense L. var. Giza 75). J Agric Food Chem, 1997, 45: 3331-3336

[6] Wang S-H(王绍华), Liu S-H(刘胜环), Wang Q-S(王强盛), Ding Y-F(丁艳锋), Huang P-S(黄丕生), Ling Q-H(凌启鸿). Relationship between yield formation and leaf nitrogen content and color in rice plant. J Nanjing Agric Univ (南京农业 大学学报), 2002, 25(4): 1-5 (in Chinese with English abstract)

[7] Wang J-H(王纪华), Huang W-J(黄文江), Zhao C-J(赵春江), Yang M-H(杨敏华), Wang Z-J(王之杰). The inversion of leaf biochemical components and grain quality indicators of winter wheat with spectral reflectance. J Remote Sens (遥感学报), 2003, 7(4): 277-284 (in Chinese with English abstract)

[8] Shiratsuchi H, Yamagishi T, Ishii R. Leaf nitrogen distribution to maximize the canopy photosynthesis in rice. Field Crops Res, 2006, 95: 291-304

[9] Qin X-D(秦晓东), Dai T-B(戴廷波), Jing Q(荆奇), Jiang $\mathrm{D}$ (姜东), Cao W-X(曹卫星). Temporal and spatial distribution of leaf nitrogen content and its relationship with plant nitrogen status in winter wheat. Acta Agron Sin (作物学报), 2006, 32(11): 1717-1722 (in Chinese with English abstract) 
[10] Ling Q-H(凌启鸿). Crop Population Quality (作物群体质量). Shanghai: Shanghai Scientific \& Technical Publishers, 2000. p 307 (in Chinese )

[11] Sun H-C(孙红春), Feng L-X(冯丽肖), Xie Z-X(谢志霞), Li C-D(李存东), Li J-C(李金才). Physiological characteristics of boll-leaf system and boll weight space distributing of cotton under different nitrogen levels. Sci Agric Sin (中国农业科 学), 2007, 40(8): 1638-1645 (in Chinese with English abstract)

[12] Xue X-P(薛晓萍), Wang J-G(王建国), Guo W-Q(郭文琦), Chen B-L(陈兵林), Wang Y-H(王友华), Zhang L-J(张丽娟), Zhou Z-G(周治国). Accumulation characteristic of biomass nitrogen and critical nitrogen concentration dilution model of cotton fruit- branch leaf after flowering. Acta Agron Sin (作物 学报), 2007, 33(4): 669-676 (in Chinese with English abstract)

[13] Williamson R E, Burn J E, Hocart C H. Towards the mechanism of cellulose synthesis. Trends Plant Sci, 2002, 7: 461-467

[14] Shu H-M(束红梅), Chen B-L(陈兵林), Wang Y-H(王友华), Hu H-B(胡宏标), Zhang W-J(张文静), Zhou Z-G(周治国). Genotypic differences in cellulose accumulation of cotton fiber and its relationship with fiber strength. Acta Agron Sin (作物学报), 2007, 33(6): 921-926 (in Chinese with English abstract)

[15] Delmer D P, Haigler C H. The regulation of metabolic flux to cellulose, a major sink for carbon in plants. Metab Eng, 2002, 4: $22-28$

[16] Haigler C H, Datcheva M I, Hogan P S, Salnikov V V, Hwang S, Kirt M, Delmer D P. Carbon partitioning to cellulose synthesis. Plant Mol Biol, 2001, 47: 29-51

[17] Amor Y, Haigler C H, Johnson S. A membrane-associated form of sucrose synthase and its potential role in synthesis of cellulose and callose in plant. Plant Biol, 1995, 92: 9353-9357

[18] Koch K. Sucrose metabolism: Regulatory mechanisms and pivotal roles in sugar sensing and plant development. Curr Opin Plant Biol, 2004, 7: 235-246

[19] Winter H, Huber S C. Regulation of sucrose metabolism in higher plants: Localization and regulation of activity of key enzymes. Crit Rev Plant Sci, 2000, 19: 31-67

[20] Michelle Babb V, Haigler C H. Sucrose-phosphate synthase activity rises in correlation with high-rate cellulose synthesis in three heterotrophic systems. Plant Physiol, 2001, 127:
$1234-1242$

[21] Zhang Z-M(张智猛), Dai L-X(戴良香), Hu C-H(胡昌浩), Dong S-T(董树亭), Wang K-J(王空军). Effects of nitrogen on starch accumulation and related enzyme activities maize. Acta Agron Sin (作物学报), 2005, 31(7): 956-962 (in Chinese with English abstract)

[22] Faleiros R R S, Seebauer J R, Below F E. Nutritionally induced changes in endosperm of shrunken 21 and brittle 22 maize kernels grown in vitrol. Crop Sci, 1996, 36: 947-954

[23] Jiang D(姜东), Yu Z-W(于振文), Li Y-G(李永庚), Yu S-L(余 松烈). Effects of different nitrogen application levels on changes of sucrose content in leaf, culm, grain and photosynthate distribution and grain starch accumulation of winter wheat. Sci Agric Sin (中国农业科学), 2002, 35(2): 157-162 (in Chinese with English abstract)

[24] Hu H-B(胡宏标), Zhang W-J(张文静), Wang Y-H(王友华), Chen B-L(陈兵林), Zhou Z-G(周治国). Matters related with cotton fiber thickening development and fiber strength. Acta Bot Boreal-Occident Sin (西北植物学报)，2007，27(4): 726-733 (in Chinese with English abstract)

[25] Li Y-K(李酉开). General Analysis Methods for Soil Agrochemistry (土壤农业化学常规分析方法). Beijing: Science Press, 1983. p 272 (in Chinese)

[26] Institute of Shanghai Plant Physiology, Chinese Academy of Sciences ed. Modern Laboratory Manual of Plant Physiology (现代植物生理学实验指南). Beijing: Science Press, 1999 (in Chinese)

[27] Konishi T, Nakai T, Sakai F, Hayashi T. Formation of callose from sucrose in cotton fiber microsomal membranes. Jpn Wood Res Soc, 2001, 47: 331-335

[28] Hikosaka K. Leaf canopy as a dynamic system: Ecophysiology and optimality in leaf turnover. Ann Bot, 2005, 95: $521-533$

[29] Liu J-H(刘继华), Yin C-Y(尹承佾), Sun Q-L(孙清荣), Wang Y-M(王永民)，Yu F-Y(于凤英). The deposition of cellulose and correlation with fiber strength in cotton. Acta Agric Nucl Sin (核农学报), 1991, 5(4): 205-209 (in Chinese with English abstract)

[30] Zhang W-J(张文静)，Hu H-B(胡宏标)，Chen B-L(陈兵林)， Shu H-M(束红梅), Wang Y-H(王友华), Zhou Z-G(周治国). Genotypic differences in some physiological characteristics during cotton fiber thickening and its relationship with fiber strength. Acta Agron Sin (作物学报), 2007, 33(4): 531-538 (in Chinese with English abstract) 